\title{
Practical View on Face Presentation Attack Detection
}

Naser Damer

naser.damer@igd.fraunhofer.de

Kristiyan Dimitrov

kristiyan.dimitrov@igd.fraunhofer.de
Smart Living \& Biometric Technologies

Fraunhofer Institute for Computer Graphics

Research (IGD)

Darmstadt, Germany
Face recognition is one of the most socially accepted forms of biometric recognition. The recent availability of very accurate and efficient face recognition algorithms leaves the vulnerability to presentation attacks as the major challenge to face recognition solutions. Previous works have shown high preforming presentation attack detection PAD solutions under controlled evaluation scenarios. This work tried to analyze the practical use of PAD by investigating the more realistic scenario of cross-database evaluation and presenting a state-of-the-art performance comparison. The work also investigated the relation between the video duration and the PAD performance. This is done along with presenting an optical flow based approach that proves to outperform state-of-the-art solutions in most experiment settings

The presented solution is tested on multiple databases: the REPLAY-ATTACK, the $M S U$ $M F S D$ and the CASIA-FASD. Each of these datasets includes subsets for training and testing to evaluate the algorithm performance.
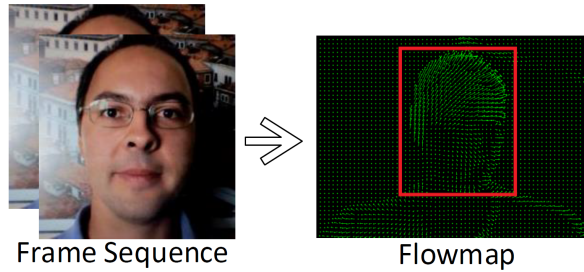

Flowmap

Figure 1: Optical flows from a face video.

The Presented PAD solution was based on optical flow in a similar manner to the Histogram of Oriented Optical Flow feature extractor (Fig.1) along with an AdaBoost classifier. Feature and score-level fusion approaches were utilized to enhance the performance.

A table of performance comparison between the proposed solution and state-of-the-art works were presented in the paper. This comparison included cross-database performance evaluation measures.

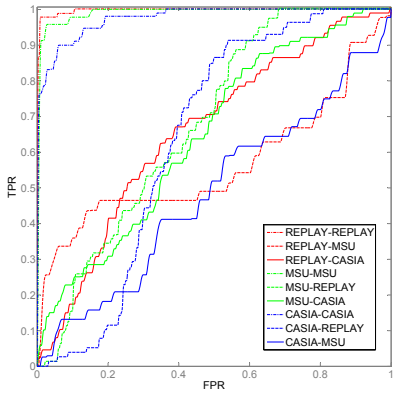

Figure 2: ROC curves achieved by the "F-triple: $\mathrm{S}$-mean" approach on intra- and cross-database evaluation.

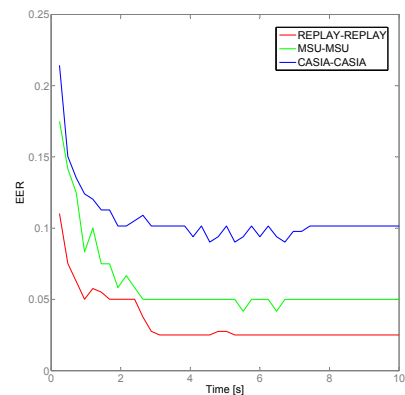

Figure 3: The performance (represented by the EER) development at different video lengths (time).

The performance degradation is clear when performing cross-database evaluation (Fig.2). However, the cross-database performance improves when considering whole videos with score-level fusion. The development of the PAD performance over the duration of the video is studied and shown in Fig.3.

To conclude, this work addressed the issue of realistic face PAD performance through evaluating it over a number of databases. This was presented along with a state-of-the-art performance comparison and a proposed solution based on optical flows. 